COLON

\title{
Suppressive effect of aspirin on aberrant crypt foci in patients with colorectal cancer
}

\author{
B Shpitz, E Klein, G Buklan, D Neufeld, A Nissan, H R Freund, M Grankin, J Bernheim
}

Gut 2003;52:1598-1601

See end of article for authors' affiliations

......................

Correspondence to: Dr B Shpitz, Department of Surgery 'B', Sapir Medical Center, Meir General Hospital, Kfar Sava, Israel 44281; shpitzca@

post.tau.ac.il

Accepted for publication 15 April 2003

\begin{abstract}
Background and aims: Aspirin and other non-steroidal anti-inflammatory drugs have been shown to reduce the risk of colorectal cancer (CRC). Animal models have shown that aspirin is also effective in reducing the density of aberrant crypt foci (ACF). The aim of the study was to evaluate the effect of chronic administration of aspirin on the distribution pattern and histological characteristics of ACF in patients with CRC.

Methods: Our study compared the distribution patterns and histomorphological characteristics of ACF between a group of CRC patients treated with low dose aspirin $(n=59)$ and a control group without aspirin $(n=135)$. ACF were visualised on methylene blue stained macroscopically normal mucosa, microdissected, and serially cut.

Results: ACF were found in $75.8 \%$ of mucosal samples from the control group and in $36 \%$ of mucosal samples from the aspirin treated group, indicating a $47 \%$ decline in prevalence of ACF in colonic samples of patients treated with aspirin. A significant reduction from $92.5 \%$ to $40 \%(p<0.0001)$ was found in distal large bowel samples containing one or more ACF. Similarly, the aspirin treated group showed a reduction in ACF density of $64 \%$ and $82 \%$, respectively, in both proximal and distal parts of the colon, indicating a significant reduction in $\mathrm{ACF} / \mathrm{cm}^{2}$ in distal colon samples $(\mathrm{p}<0.01)$. The aspirin treated group displayed a $52 \%$ reduction in dysplastic ACF although this difference was not statistically significant.

Conclusions: Our study has provided evidence of the effective chemopreventive action of low dose aspirin on ACF in humans.
\end{abstract}

olorectal cancer (CRC) is one of the most common malignancies of the aging population in Western countries and the third leading cause of cancer deaths in the USA. ${ }^{1}$ Epidemiological studies have shown that nonsteroidal anti-inflammatory drugs (NSAIDs) reduce the risk of colorectal adenomas and carcinomas. Furthermore, large scale epidemiological studies indicate that CRC related deaths were decreased by $40-50 \%$ in patients who were treated with aspirin for long periods of time. ${ }^{2-4}$ There are almost no data however on the effect of aspirin on early stages of colorectal tumorigenesis in humans.

Aberrant crypt foci (ACF) are considered one of the earliest preneoplastic and early neoplastic lesions in the multistep process of colorectal tumorigenesis. Abundant clinical and laboratory data link the lesions with early steps of neoplastic progression..$^{5-11}$ ACF are present with high frequency in patients with $\mathrm{CRC}^{589^{11}}$ and carry molecular alterations characteristic for early colonic neoplasia. ${ }^{10}$ Thus ACF are considered a potential biomarker for CRC risk.

The effect of NSAIDs on early precursors of CRC has been studied mainly in animal models. Numerous animal studies have evaluated the effect of aspirin and other NSAIDs on ACF and colon cancer. ${ }^{6}{ }^{12-15}$ Most studies have demonstrated a significant reduction in total ACF per colon in rats treated with sulindac, aspirin, and other NSAIDs. ${ }^{12} 131617$ In humans, ACF were rarely found in those who received NSAIDs for more than one year. ${ }^{10}$ Furthermore, a prospective follow up of patients treated with sulindac showed a significant reduction in the number of ACF while numbers remained mostly unchanged in control patients who did not receive sulindac. ${ }^{10}$ This study has provided the first evidence of an NSAID effect during the early stages of colonic carcinogenesis in humans. To the best of our knowledge, there are no data on the suppressive effect of aspirin on ACF in humans or its effect on histological types of ACF.
The objective of our study was to evaluate the effect of chronic administration of aspirin on the distribution pattern and histological characteristics of ACF in a group of patients with CRC.

\section{MATERIALS AND METHODS Study group}

Normal appearing colorectal mucosal samples were collected from surgical resection specimens obtained from 194 patients who underwent large bowel resection for sporadic CRC. Patients with familial syndromes of CRC (familial adenomatoid polyposis and hereditary non-polyposis colon cancer), patients on NSAID treatment other than aspirin, as well as those who received preoperative radiation and/or chemotherapy were excluded from the study. Information regarding administration of drugs other than aspirin or NSAIDs and history of accompanying diseases were available in $50 \%$ of patients. Therefore, these details were not included in the data analysis. Dietary habits of the patients were not available. Mucosal samples were collected at least $2 \mathrm{~cm}$ from the tumour margin. Mean size of the dissected mucosal surface was 64.2 (16) $\mathrm{cm}^{2}$ (range 45-116 $\mathrm{cm}^{2}$ ).

Fifty nine patients had been receiving aspirin treatment on a regular basis for at least one year.

Tissue collection, sampling, and processing For identification and collection of ACF, mucosal samples were spread flat and fixed in $4 \%$ buffered

Abbreviations: ACF, aberrant crypt foci; CRC, colorectal cancer; NSAIDs, non-steroidal anti-inflammatory drugs; PPAR, peroxisome proliferator activated receptor 

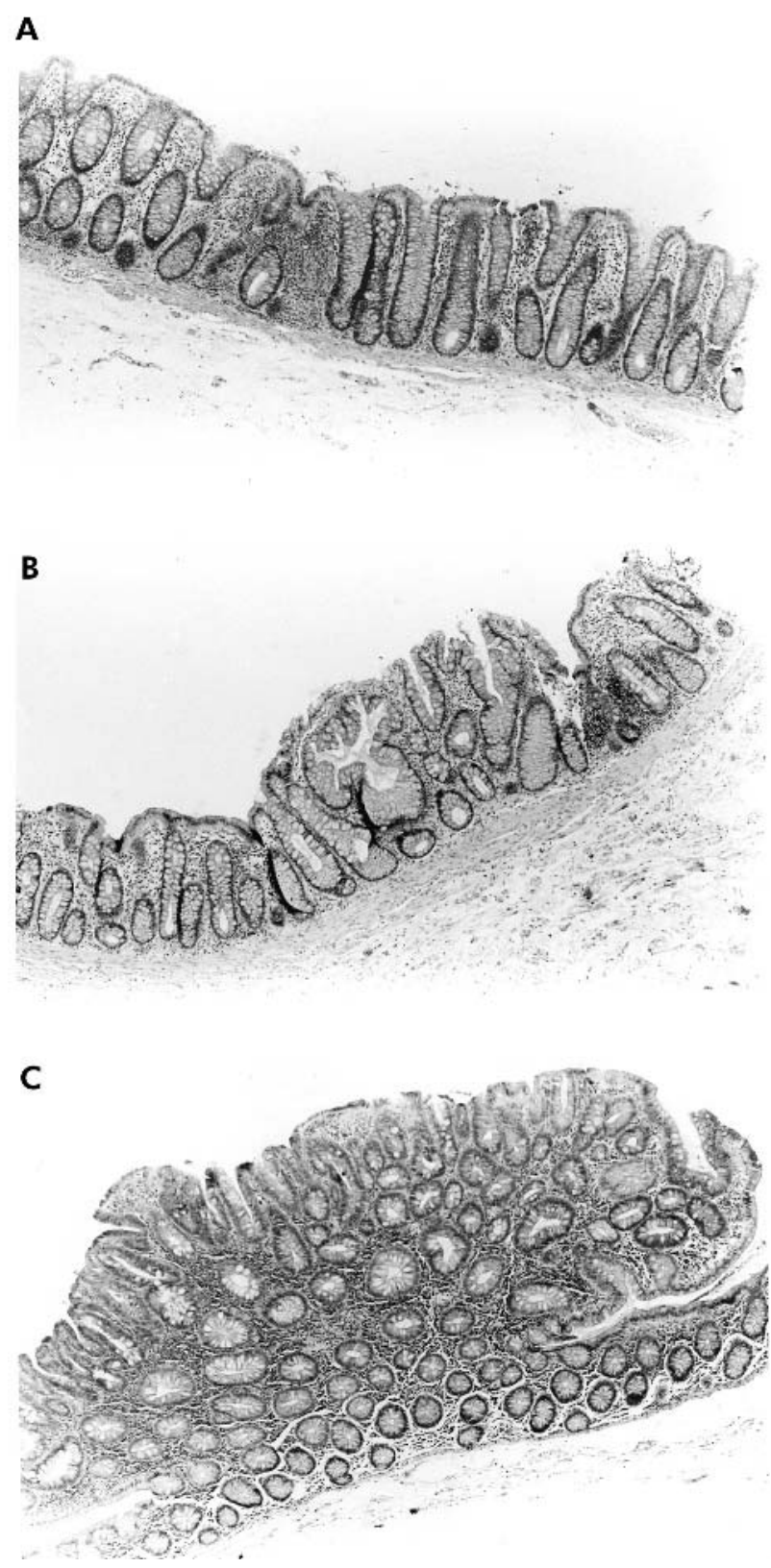

Figure 1 Histological sections of aberrant crypt foci (ACF). ACF were dissected along the crypts, perpendicular to the mucosal surface. (A) Non-hyperplastic non-dysplastic ACF; (B) hyperplastic ACF; and (C) dysplastic ACF (haematoxylin-eosin, magnification $\times 200$ ).

formalin. ACF were screened under low power magnification following staining in $1 \%$ methylene blue. The identified ACF were counted, microdissected, embedded in paraffin, sectioned, and stained with haematoxylineosin. According to the histopathological pattern, ACF were classified as non-hyperplastic, hyperplastic, and dysplastic (fig 1). ${ }^{18}$ A total of 291 ACF were microdissected.

Data analysis included comparison of the prevalence, distribution density, multiplicity (number of foci per ACF), and histopathological characteristics of ACF in patients with and without aspirin treatment. The distribution pattern of ACF in both groups was compared separately for the proximal and distal colon. The anatomical boundary between the distal and proximal parts of the large bowel was the splenic flexure.
Table 1 Duration of aspirin administration in the treatment group

\begin{tabular}{ll}
\hline Time on aspirin (months) & No of patients (\%) \\
\hline $12-60$ & $35(59.3)$ \\
$61-120$ & $21(35.6)$ \\
$>120$ & $3(5.1)$ \\
\hline
\end{tabular}

\section{Statistical analysis}

Comparison between groups was performed with the MannWhitney U test and the $\chi^{2}$ test, as appropriate, using the SPSS statistical package (SPSS Inc, Chicago, Illinois, USA). All values are expressed as mean (SD), unless indicated otherwise.

\section{RESULTS}

A total of 206 mucosal samples were obtained from 194 patients. More than one specimen was obtained from some patients who underwent subtotal large bowel resection. Fifty nine patients had been receiving aspirin treatment for at least one year. All patients in the study group were treated with $100 \mathrm{mg} /$ day aspirin except for three who were treated with buffered aspirin $325 \mathrm{mg} /$ day. Average time on aspirin was 48 months (range 12-108 months). Data on duration of aspirin treatment are shown in table 1.

There were 65 (48\%) women and $70(52 \%)$ men in the control group and nine (16\%) women and 50 (84\%) men in the study (aspirin) group. Mean ages of the control and study groups were 72.6 (range 35-86) years and 69.6 (range 62-82) years, respectively.

Baseline ACF density in the control group was calculated separately for the right and left colons. No significant difference in ACF density was found between the different age groups (table 2). ACF were found in $75.8 \%$ of mucosal samples from the control group and in $36 \%$ of those from aspirin treated patients, indicating a $47 \%$ decline in prevalence of ACF in "treated" colonic samples. There was a reduction in samples that contained at least one ACF from $46 \%$ to $32 \%$ in the proximal $(p=0.9)$ and from $92.5 \%$ to $40 \%$ $(\mathrm{p}<0.0001)$ in the distal large bowel (table 3$)$.

The distribution density of ACF in the control and aspirin treated groups is shown in table 4 . The aspirin treated group showed a reduction of $64 \%$ and $82 \%$ in ACF density in both proximal and distal parts of the colon, respectively, indicating a significant reduction in $\mathrm{ACF} / \mathrm{cm}^{2}$ in the distal but not in the proximal colon samples $(p=0.09$ for the proximal colon; $\mathrm{p}<0.01$ for the distal colon) (table 4 ).

The effect of aspirin on the histological characteristics of microdissected ACF is shown in table 5. Aspirin treatment resulted in a redistribution pattern of dysplastic ACF: the aspirin treated group displayed a $52 \%$ reduction in dysplastic ACF compared with the control group, although this difference was not statistically significant (table 5).

Table 2 Density of aberrant crypt foci (ACF) in different age groups in the control group (expressed as $A C F / \mathrm{cm}^{2}$ )

\begin{tabular}{llllll}
\hline \multirow{5}{*}{ Site of colon } & \multicolumn{4}{l}{ Age group $(y)$} \\
\cline { 2 - 6 } & $<50$ & $51-60$ & $\mathbf{6 1 - 7 0}$ & $\mathbf{7 1 - 8 0}$ & $>80$ \\
\hline Right & 0.04 & 0.04 & 0.01 & 0.02 & 0 \\
Left & 0.12 & 0.09 & 0.12 & 0.1 & 0.16 \\
\hline
\end{tabular}


Table 3 Effect of aspirin on overall prevalence of aberrant crypt foci (ACF) in colonic samples

\begin{tabular}{llllll}
\hline & \multicolumn{2}{l}{ Control group } & & \multicolumn{2}{l}{ Aspirin group } \\
\cline { 6 - 6 } & Right & Left & & Right & Left \\
\hline Total No of samples & 47 & 94 & & 25 & 40 \\
No $(\%)$ of samples without ACF & $25(54)$ & $7(7.5)$ & & $17(68)$ & $24(60)$ \\
No $(\%)$ of samples with ACF & $22(46)$ & $87(92.5)$ & & $8(32)$ & $16(40)$ \\
\hline
\end{tabular}

Table 4 Density of aberrant crypt foci (ACF) in the control and aspirin treated groups

\begin{tabular}{llll}
\hline & \multicolumn{2}{l}{ Density of $\mathrm{ACF}\left(\mathrm{ACF} / \mathrm{cm}^{2}\right)$} & \\
\cline { 2 - 3 } & Control group & Aspirin group & \% reduction in density of ACF (p value*) \\
\hline Right colon & $0.047(0.12)$ & $0.017(0.03)$ & $63(0.09)$ \\
Left colon & $0.13(0.12)$ & $0.024(0.02)$ & $72(<0.01)$ \\
\hline
\end{tabular}

Values are mean (SD). *Mann-Whitney $U$ test.

\section{DISCUSSION}

Our findings showed that low dose aspirin treatment, administered for at least one year before surgery, resulted in reduction of overall density of ACF. We further demonstrated that significantly fewer mucosal samples in the aspirin treated group contained ACF. This is the first study in humans to demonstrate a chemopreventive effect of low dose aspirin at the earliest stages of colon tumorigenesis.

Aspirin plays a major role in primary and secondary prevention of cardiovascular events such as myocardial infarction and cerebrovascular accidents. ${ }^{19-21}$ It is therefore not surprising that a substantial percentage of elderly patients with CRC take aspirin as a chemopreventive agent for cardiovascular events. This fact has enabled us to collect prospective data on a group of patients who had been receiving chronic aspirin treatment and to evaluate the effect of aspirin on the distribution and histological characteristics of ACF by comparing them with a group of patients who were not taking aspirin. The design of our study warrants special comment. Patients in our study group had already had CRCs, giving rise to concerns that many of the patients might be resistant to the chemopreventive action of aspirin. This could be relevant, at least for those patients who had been taking aspirin for a long period of time (longer than a decade). In fact, the median time on aspirin in our study group was relatively short (48 months) and only a small proportion of our patients $(5 \%)$ had been taking aspirin for more than 10 years. Although Greenberg and colleagues demonstrated a lower risk for adenomas even after one year on aspirin treatment, ${ }^{22}$ another study found that a substantial reduction in CRC risk could be demonstrated only after at least 10 years of aspirin use. ${ }^{23}$ Interestingly, our results indicate that even among patients with CRC, aspirin treatment was capable of exerting suppressive action at the earliest stages of colorectal tumorigenesis.

Two factors that could have influenced the distribution patterns, prevalence, and histological subtypes of ACF in our study were diet and medications. Different dietary components have been shown to affect the prevalence of ACF in carcinogen induced colon tumorigenesis models in rodents. High fat diets containing mixed lipids ${ }^{24}$ increased the total number of ACF while high energy and fat restricting diets exerted a suppressive effect on advanced ACF. ${ }^{25}$ Similarly, dietary supplementation of wheat germ extract ${ }^{26}$ and fermented brown rice ${ }^{27}$ effectively inhibited ACF development. However, as no studies have been conducted in humans to evaluate the possible effects of various dietary components on ACF, the potential effect of diet is largely unknown. Data on dietary restrictions were not available in our patients with cardiovascular diseases; therefore, although theoretically a low fat diet recommended for patients with cardiovascular diseases could influence the distribution patterns of ACF, the real influence of these components on the prevalence of ACF in our study remains unknown. Similarly, no studies in humans regarding the effect on ACF of cardiovascular or other medications, except NSAIDs, have been reported in the literature.

Aspirin and other NSAIDs were shown to be effective chemopreventive agents during the initiation and postinitiation stages of colonic carcinogenesis in rodents. ${ }^{12-14} 2829$ Low dose aspirin was effective in suppressing ACF formation but was unable to prevent cancer..$^{1328}$ No specific data from these studies have demonstrated how aspirin specifically affected dysplastic ACF. To the best of our knowledge no studies have been published on the effect of aspirin on early morphological biomarkers of colonic carcinogenesis in humans. A recent study using magnification colonoscopy evaluated the effect of sulindac on ACF and showed that this agent was indeed effective in reducing the density and prevalence of ACF in the rectum, one year after initiation of treatment. ${ }^{10}$ Although this study demonstrated complete disappearance of ACF in seven of 11 treated patients and reduction of ACF density in the rest, no specific data on dysplastic foci were provided. As only one patient in this group actually had colon cancer, it was probably unreliable to search specifically for the effect of sulindac on dysplastic lesions.

Even though we were able to demonstrate a general ACF suppressive effect, the more critical issue in evaluation of chemopreventive action of low dose aspirin would be the potential effect specifically on dysplastic ACF which are truly

Table 5 Effect of aspirin on histological types of aberrant crypt foci (ACF)

\begin{tabular}{lccc}
\hline \multirow{2}{*}{ Histology of ACF } & Control group & & Aspirin group \\
\cline { 2 - 2 } & No (\%) of ACF & & No (\%) of ACF \\
\hline Dysplastic* & $32(13.3)^{*}$ & $4(6.9)^{*}$ \\
Hyperplastic & $147(61.2)$ & $34(58.6)$ \\
Non-D, non-H & $61(25.5)$ & $21(34.5)$ \\
\hline${ }^{*} N S$ ( ${ }^{2}$ test). & & \\
\hline
\end{tabular}


neoplastic microscopic lesions and have been recognised as the most important lesions in the ACF-adenoma-carcinoma sequence. While we were able to demonstrate a general ACF suppressing effect, our data showed that although aspirin treated patients displayed a reduced proportion of dysplastic ACF (from $13.3 \%$ in the control group to $6.3 \%$ in treated patients), the results were not statistically significant. Surprisingly, there are no data addressing this important issue.

The vast majority of our patients in the aspirin group had been on low dose aspirin. The minimal effective aspirin dose in early colonic tumorigenesis is unknown. Ruffin et al suggested that a single $81 \mathrm{mg}$ dose of aspirin taken daily should be sufficient to significantly reduce colorectal mucosal prostaglandins $\mathrm{E}_{2}$ and $\mathrm{F}_{2} \cdot{ }^{30}$ Based on these data, the above dose was recommended for future chemopreventive studies in CRC. Although an aspirin dose close to that reported in the latter study could explain the effect of aspirin on ACF observed in our study, it is not yet known whether this dose would be sufficient to suppress colorectal carcinogenesis.

Our present study showed that a greater reduction in ACF density was noted in the distal compared with the proximal colon. In fact, the only statistically significant reduction in the percentage of samples containing ACF as well as a reduction in ACF density was found in the distal large bowel. This is in accordance with findings in a previous study in a rodent model $^{31}$ that demonstrated a distal predilection of nabumetone suppressive action on ACF. The cause of this differential suppression of ACF in different parts of the large bowel is unclear although higher levels of cyclooxygenase 2 expression as well as stronger induction of peroxisome proliferator activated receptor (PPAR) in distal parts of premalignant rat colons have been found. ${ }^{31}$ It is noteworthy that both PPAR and cyclooxygenase 2 are molecular targets of NSAIDs. ${ }^{32-34}$

In conclusion, this study has provided the first evidence of a chemopreventive effect of low dose aspirin on ACF in humans. Further studies should be carried out to evaluate the potential mechanisms of the differential effect of aspirin and other NSAIDs on ACF in humans as well as their effect on dysplastic ACF.

\section{ACKNOWLEDGEMENTS}

Supported by Israel Cancer Association Grant.

\section{Authors' affiliations}

B Shpitz, E Klein, G Buklan, D Neufeld, Department of Surgery, Sapir Medical Center, Meir General Hospital, Kfar Sava, Tel Aviv University Sackler School of Medicine, Israel

A Nissan, H R Freund, Department of Surgery, Hebrew UniversityHadassa Medical School, Israel

M Grankin, Department of Biostatistics, Sapir Medical Center, Meir General Hospital, Kfar Sava, Tel Aviv University Sackler School of Medicine, Israel

J Bernheim, Department of Pathology, Sapir Medical Center, Meir General Hospital, Kfar Sava, Tel Aviv University Sackler School of Medicine, Israel

\section{REFERENCES}

1 Cancer statistics 2002. CA Cancer J Clin 2002;52:23-47.

2 Giovancucci E. The prevention of colorectal cancer by aspirin use. Biomed Pharmacother 1999:53:303-8.

3 Jänne PA, Mayer RJ. Chemoprevention of colorectal cancer. N Engl J Med 2000;342:1960-8.

4 Shaheen NJ, Straus WL, Sandler RS. Chemoprevention of gastrointestinal malignancies with nonsteroidal antiinflammatory drugs. Cancer 2002;94:950-63.
5 Pretlow TP, Barrow BJ, Ashton WS, et al. Aberrant crypts: putative preneoplastic foci in human colonic mucosa. Cancer Res 1991;51:1564-7.

6 Wargovich MJ, Jimenez A, McKee K, et al. Efficacy of potential chemopreventive agents on rat colon aberrant crypt formation and progression. Carcinogenesis 2000;21:1149-55.

7 Roncucci L, Pedroni M, Vaccina F, et al. Aberrant crypt foci in colorectal carcinogenesis. Cell Prolif 2000;33:1-18.

8 Roncucci L, Stamp D, Medline A, et al. Identification and quantification of aberrant crypt foci and microadenomas in human colons. Hum Pathol $1991 ; 22: 287-94$

9 Smith AJ, Stern HS, Penner M, et al. Somatic APC and K-ras codon 12 mutations in aberrant crypt foci from human colons. Cancer Res 1994;54:5527-30.

10 Takayama T, Katsuki S, Takahashi Y, et al. Aberrant crypt foci of the colon as precursors of adenoma and cancer. N Engl J Med 1998;339:1277-84.

11 Shpitz B, Bomstein Y, Mekori Y, et al. Aberrant crypt foci in human colons: distribution and histopathologic characteristics. Hum Pathol 1998;29:469-75

12 Wargovich MJ, Chen CD, Harris C, et al. Inhibition of aberrant crypt growth by non-steroidal anti-inflammatory agents and differentiation agents in the rat colon. Int J Cancer 1995;60:515-19.

13 Shpitz B, Bomstein Y, Kariv N, et al. Chemopreventive effect of aspirin on growth of aberrant crypt foci in rats. Int J Colorect Dis 1998;13:169-72.

14 Barnes CJ, Hardman E, Cameron IL, et al. Aspirin, but not sodium salicylate, indomethacin or nabumetone reversibly suppresses 1,2-dimethylhidrazineinduced colonic aberrant crypt foci in rats. Dig Dis Sci 1997;42:920-6.

15 Bak AW, McKnight W, Li P, et al. Cyclooxygenase-independent chemoprevention with an aspirin derivative in a rat model of colonic adenocarcinoma. Life Sci 1998;62:PL367-73.

16 Reddy BS, Rao CV, Rivenson A, et al. Inhibitory effect of aspirin on azoxymethane-induced colon carcinogenesis in F344 rats. Carcinogenesis 1993; 14:1493-7.

17 Mereto E, Frencia L, Ghia M. Effect of aspirin on incidence and growth of aberrant crypt foci induced in the rat colons by 1,2-dimethylhidrazine. Cancer Lett 1994:76:5-9.

18 Di Gregorio C, Losi L, Fante R, et al. Histology of ACF in human colons. Histopathology 1997;30:328-34.

19 Sanmuganathan PS, Ghahramani P, Jackson PR, et al. Aspirin for primary prevention of coronary heart disease: safety and absolute benefit related to coronary risk derived from meta-analysis of randomized trials. Heart 2001;85:265-71.

20 Calverley DC. Thromboembolic disease and anticoagulation in the elderly. Clin Geriatr Med 2001;17:31-48.

21 International Stroke Collaborative Group. The international stroke trial (IST): a randomized trial of aspirin, subcutaneous heparin, both or neither, among 19435 patients with acute ischaemic stroke. Lancet 1997;349:1569-81.

22 Greenberg ER, Baron JA. Reduced risk of large bowel adenomas among aspirin users. The Polyp Prevention Study Group. J Natl Cancer Inst 1993;85:912-16.

23 Giovancucci E, Rimm EB, Stampfer MJ, et al. Aspirin use and the risk of colorectal cancer and adenoma in male health professionals. Ann Intern Med 1994; 121:241

24 Rao CV, Hirose Y, Indranie C, et al. Modulation of experimental colon tumorigenesis by types and amounts of dietary fatty acids. Cancer Res 2001:61:1927-33.

25 Lasko CM, Good CK, Adam J, et al. Energy restriction modulates the development of advanced preneoplastic lesions depending on the level of fat in the diet. Nutr Cancer 1999;33:69-75.

26 Zalantai A, Lapis K, Szende B, et al. Wheat germ extract inhibits experimental colon carcinogenesis in F-344 rats. Carcinogenesis 2001;22:1649-52.

27 Katayama M, Yoshimi N, Yamada Y. Preventive effect of fermented brown rice and rice bran against colon carciogenesis in male F344 rats. Oncol Rep 2002;9:817-22.

28 Pereira MA. Prevention of colon cancer and modulation of aberrant crypt foci, cell proliferation, and apoptosis by retinoids and NSAIDs. Adv Exp Med Biol 1999:470:55-63.

29 Li H, Schut HA, Conran P, et al. Prevention by aspirin and its combination with alfa-difluoromethylornitine of azoxymethane-induced tumors, aberrant crypt foci and prostaglandin $\mathrm{E}_{2}$ levels in rat colon. Carcinogenesis 1999:20:425-30.

30 Ruffin IV MT, Krishnan K, Rock CL, et al. Suppression of human colorectal mucosal prostaglandins: determining the lowest effective aspirin dose. J Natl Cancer Inst 1997;89:1 152-60.

31 Roy HK, Karolski WJ, Ratashak A. Distal bowel selectivity in the chemopreventionn of experimental colon carcinogenesis by the non-steroidal anti-inflammatory drug nabumetone. Int J Cancer 2001 ;92:609-15.

32 Shiff SJ, Rigas B. The role of cyclooxygenase inhibition in the antineoplastic effect of nonsteroidal antiinflammatory drugs (NSAIDs). J Exp Med 1999; 190:445-50

33 He TC, Chan TA, Vogelstein B, et al. PPARdelta is an APC-regulated target of nonsteroidal anti-inflammatory drugs. Cell 1999;99:335-45.

34 Ota S, Bamba H, Kato A, et al. COX-2, prostanoids and colon cancer. Alliment Pharmacol Ther 2002;16(suppl 2):102-6. 